\title{
Kosten-Nutzen-Analyse für ein Regionalspital
}

\author{
E. Kilgus*, H.-P. Simmen
} * em. Professor für Betriebswirtschafts-
lehre, Universität Zürich

Korrespondenz:

Prof. Dr. med. Hans-Peter Simmen

Spital Oberengadin

Chirurgische Abteilung

CH-7503 Samedan
Ein allen vertrautes Bild: Gegeben ist ein gut geführtes und deshalb geschätztes Regionalspital, das Jahr für Jahr mit seiner Ärzteschaft, seinem Pflegedienst und seinen weiteren Mitarbeitern unentbehrliche Dienste erbringt, aber dessen ungeachtet nicht in der Lage ist, mit den zufliessenden Erlösen alle anfallenden Kosten $\mathrm{zu}$ decken. Standortkanton und Standortgemeinden sind deshalb gehalten, alle resultierenden Verluste abzudecken.

Dies gilt auch für das Spital Oberengadin in Samedan. Der Kanton Graubünden und die elf Kreisgemeinden Bever, Celerina, Madulain, La Punt-Chamues-ch, Pontresina, Samedan, S-chanf, St. Moritz, Sils, Silvaplana und Zuoz teilen sich im Verhältnis von etwa $2 / 3 \mathrm{zu}^{1 / 3}$ in die Deckung des Jahresverlustes von etwa 7 Millionen Franken. So kann es nicht erstaunen, dass von Zeit zu Zeit die Frage aufgeworfen wird, ob sich denn die Führung eines solchen Spitals mit rund 300 Mitarbeitern und gegen 12000 Patienten überhaupt lohne. Stets werden Wege zur Verbesserung der Wirtschaftlichkeit gesucht, mit der Schaffung eines Spitalverbundes Südbünden oder anderen Formen der Schwergewichtsbildung.

Vor diesem Hintergrund hat die Spitalkommission am 1. Mai 2000 eine interdisziplinäre Arbeitsgruppe beauftragt, eine regionale Wertschöpfungsanalyse durchzuführen, welche den Stellenwert des Spitals für das Oberengadin anhand sorgfältiger Analysen aufzeigen soll.

Der Bericht liegt vor und das Ergebnis sei vorweggenommen: Die Untersuchung hat ergeben, dass dem Jahresverlust von etwa 7 Millionen Franken eine Wertschöpfung in der Höhe von 15 Millionen Franken gegenübersteht. Dazu beigetragen haben die Mitarbeiter des Spitals mit 11 Millionen Franken, die Patienten mit 3 Millionen Franken und das Spital selbst mit 1 Million Franken. Zu beantworten ist die Frage, wie sich denn ein solcher Wertschöpfungsbeitrag überhaupt errechnet.

Wie angedeutet, hat die Arbeitsgruppe drei voneinander unabhängige Teiluntersuchungen veranlasst.

Die erste Teiluntersuchung, die Analyse über den Spitalbetrieb, erfasst die spitaleigene Nachfrage nach Gütern und Dienstleistungen bei 99 Zulieferbetrieben aus der Region. Gemessen wurden dabei die vom Spital ausgelösten Arbeitseinkommen, die Zahl der dank des Spitals kreierten Arbeitsplätze und die Steuerabgaben der betroffenen Unternehmen und Mitarbeiter der Zulieferer. Gefragt wurden die 28 wichtigsten der 99 erwähnten Betriebe. Für die Interviews stellte sich eine Abschlussklasse einer Mittelschule in Samedan (Academia Engiadina) zur Verfügung. Die regionale Wertschöpfung der Zulieferbetriebe belief sich auf etwa 1 Million Franken.

Die zweite Teiluntersuchung umfasst über eine schriftliche Befragung die Gesamtheit der Spitalmitarbeiter. Die Vollerhebung bezog sich auf die Einkommensverwendung, wie Konsum, Sparverhalten und Einkommenssteuern, getrennt nach den 11 Wohnsitzgemeinden. Der Wertschöpfungsbeitrag der Mitarbeiter ergab 11 Millionen Franken.

Die dritte Teiluntersuchung erfolgte im Kreise der Patienten. Ermittelt wurde die Nachfrage der Patienten nach den Dienstleistungen des Spitals und des Gast- und Tourismusgewerbes im Umfeld. Im Mittelpunkt standen die Tagesausgaben der Patienten bzw. ihrer begleitenden oder besuchenden Angehörigen mit anfallenden Verkehrskosten, getrennt nach den vier Kategorien «Gäste-Patienten» (vorwiegend aus Italien, «Gäste-Angehörige», «einheimische Patienten» und «einheimische Angehörige»). 500 Personen wurden in die durch das Spitalpersonal durchgeführte Erhebung einbezogen. Der Wertschöpfungsbeitrag belief sich auf über 3 Millionen Franken.

Wie erwähnt, ergibt sich aus den drei voneinander unabhängigen Analysen ein totaler Wertschöpfungsbeitrag von rund 15 Millionen Franken. Unter Beachtung aller möglichen Fehlerquellen, die sich durch die Befragungstechnik und die Beschränkung gewisser Untersuchungen auf Teilerhebungen (Stichprobenfehler) ergeben können, geht man nicht fehl in der Annahme, dass die rund 7 Millionen Franken der staatlichen Defizitdeckung durch die spitalbedingte Wertschöpfung von rund 15 Millionen Franken in die regionale gesamtwirtschaftliche Rechnung zurückgeflossen sind. Interessant ist dabei, dass die alleine von den Spitalmitarbeitern in ihren Wohnortgemeinden abgelieferten Einkommenssteuern teilweise höher waren als die Beiträge dieser Gemeinden zur Verlustdeckung des Spitals (insbesondere bei der Standortgemeinde Samedan). 
Die mit der Erhebung verbundenen theoretisch-methodischen und praktischen Probleme sind in den Studien des Instituts für Tourismus und Landwirtschaft der Academia Engiadina am 1. Juli 2001 als Band 1 der neuen Schriftenreihe veröffentlicht worden und können jederzeit eingesehen werden (auch im Internet unter www.kreisamt-oberengadin.ch $\rightarrow$ Kreis/Übersicht $\rightarrow$ Gesundheit und Alter $\rightarrow$ Spital Oberengadin). Die Publikation zeigt auch, wie wichtig es ist, dass sich die Ärzteschaft und die Führungsund Leitungsorgane des Spitals hinter ein solches Projekt stellen, da es eine wertvolle Entscheidungshilfe für die Behörden aller Stufen darstellt. 